\title{
Chemical fractionation in systems condensing from the gas phase
}

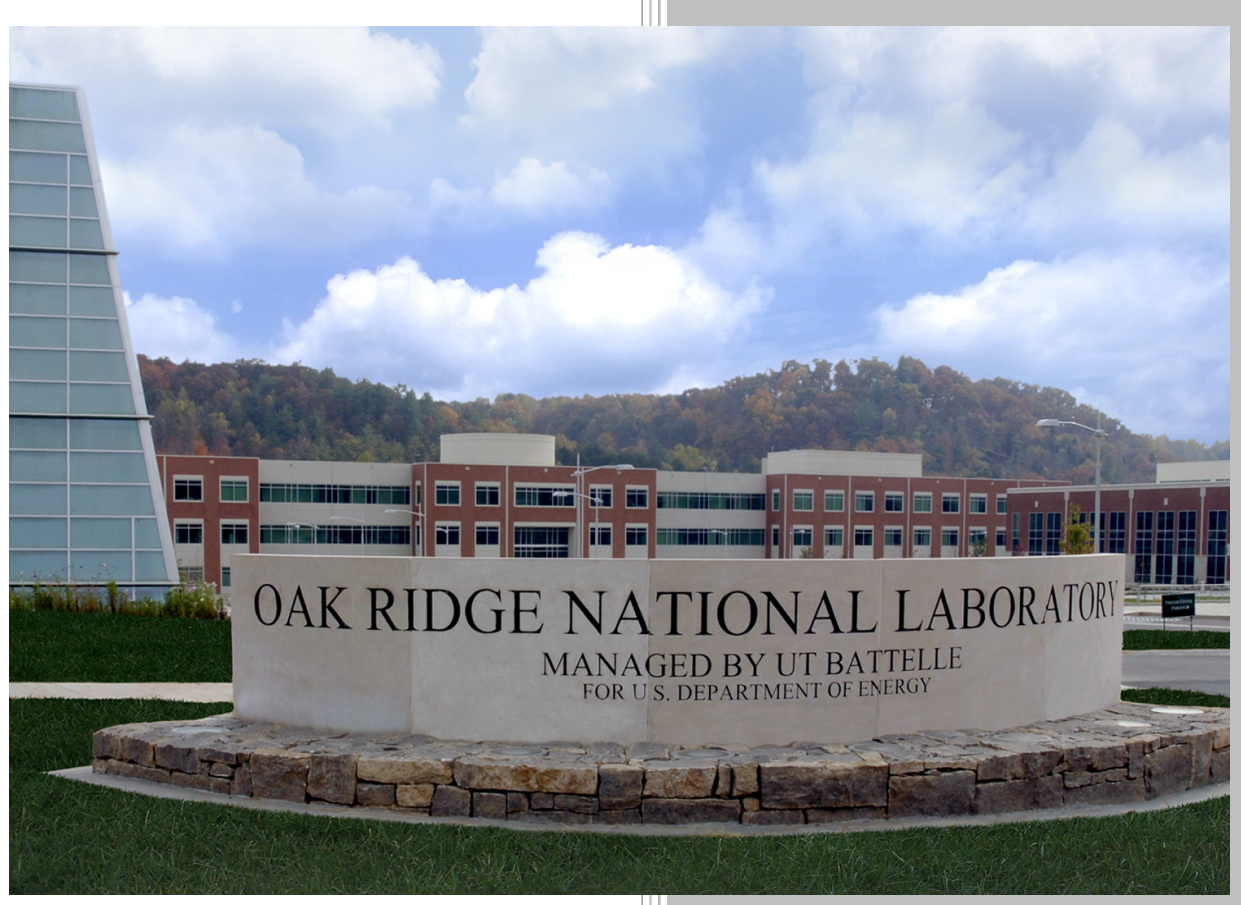

\section{Approved for public release.} Distribution is unlimited.

Pablo Moresco

January 2021 


\section{DOCUMENT AVAILABILITY}

Reports produced after January 1, 1996, are generally available free via US Department of Energy (DOE) SciTech Connect.

Website: www.osti.gov/

Reports produced before January 1, 1996, may be purchased by members of the public from the following source:

National Technical Information Service

5285 Port Royal Road

Springfield, VA 22161

Telephone: 703-605-6000 (1-800-553-6847)

TDD: $703-487-4639$

Fax: 703-605-6900

E-mail: info@ntis.gov

Website: http://classic.ntis.gov/

Reports are available to DOE employees, DOE contractors, Energy Technology Data Exchange representatives, and International Nuclear Information System representatives from the following source:

Office of Scientific and Technical Information

PO Box 62

Oak Ridge, TN 37831

Telephone: 865-576-8401

Fax: 865-576-5728

E-mail: report@osti.gov

Website: http://www.osti.gov/contact.html

This report was prepared as an account of work sponsored by an agency of the United States Government. Neither the United States Government nor any agency thereof, nor any of their employees, makes any warranty, express or implied, or assumes any legal liability or responsibility for the accuracy, completeness, or usefulness of any information, apparatus, product, or process disclosed, or represents that its use would not infringe privately owned rights. Reference herein to any specific commercial product, process, or service by trade name, trademark, manufacturer, or otherwise, does not necessarily constitute or imply its endorsement, recommendation, or favoring by the United States Government or any agency thereof. The views and opinions of authors expressed herein do not necessarily state or reflect those of the United States Government or any agency thereof. 
Nuclear Nonproliferation Division

\title{
CHEMICAL FRACTIONATION IN SYSTEMS CONDENSING FROM THE GAS PHASE
}

\author{
Pablo Moresco
}

Date Published: January 2021

Prepared by

OAK RIDGE NATIONAL LABORATORY

Oak Ridge, TN 37831-6283

managed by

UT-Battelle, LLC

for the

US DEPARTMENT OF ENERGY

under contract DE-AC05-00OR22725 



\section{CONTENTS}

LIST OF FIGURES $\ldots \ldots \ldots \ldots \ldots \ldots \ldots \ldots \ldots \ldots \ldots \ldots \ldots \ldots$

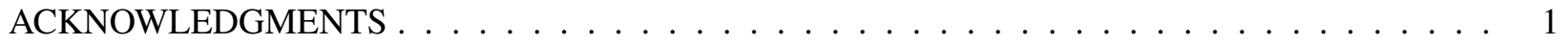

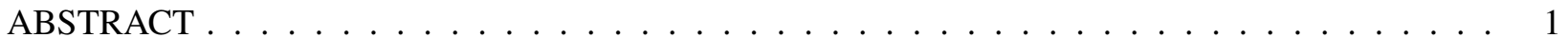

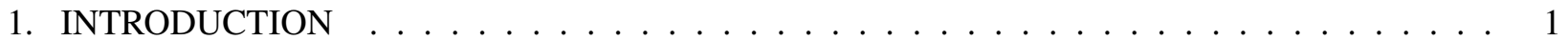

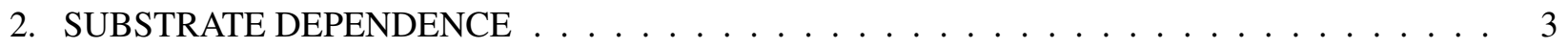

3. EVOLUTION OF THE CONDENSED PHASE $\ldots \ldots \ldots \ldots \ldots \ldots \ldots \ldots$

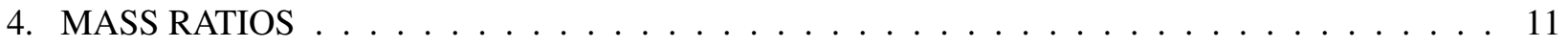

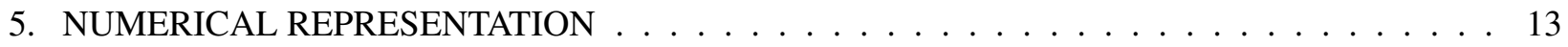

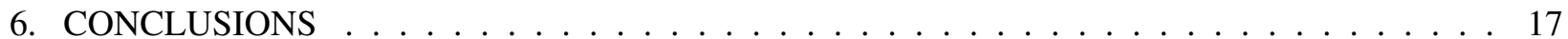

7. REFERENCES . . . . . . . . . . . . . . . . . . . . . . . . . . 19 



\section{LIST OF FIGURES}

1 Differences in the normalized mass distribution of components of a particle population are an indication of fractionation. . . . . . . . . . . . . . . . 2

2 Mass fluxes as a function of particle radius and Knudsen number $(\mathrm{Kn})$ for a $\mathrm{FeO}$ vapor in air at 2,000 K. . . . . . . . . . . . . . . . . . . . . . . . 4

3 Magnitude of the collision kernel for particles with density $5.8 \mathrm{~g} / \mathrm{cm}^{3}$ in air at $2,000 \mathrm{~K}$ of (a) a particle of radius $10^{-8} \mathrm{~m}$ and (b) a particle with radius $10^{-6} \mathrm{~m} . \ldots \ldots$

4 Schematic representation of the number, radius, surface and volume distributions for a particle population (not to scale) . . . . . . . . . . . . . . 5

5 Condensation of $B$ on $A$ particles when the substances are not miscible (a) and when they

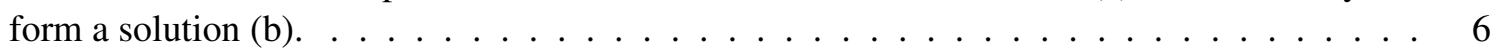

6 Schematic representation of the mass distributions of species $A$ and $B$ when they are miscible (a) and when they are not (b) . . . . . . . . . . . . . . . 7

7 Particle characteristics when $A$ and $B$ are immiscible when coagulation is weak (a) and

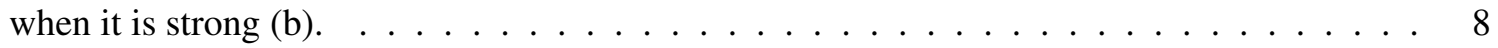

8 Variations in particle characteristics as a function of the solubility of $C$ in $B$, when $A$ and $B$ are not miscible. . . . . . . . . . . . . . . . . . . 8

9 Schematic representation of the species mass distributions corresponding to Figure 8 . . . . 9

10 Particle characteristics and species mass distributions as a function of the solubility of $C$ in $B$, when $A$ and $B$ are miscible. . . . . . . . . . . . . . . . . . . 9

11 Fraction of the total mass in the condensed phase as a function of time for each species (left), and volume fraction of the condensed phase normalized for its value at time 10 seconds (right). These curves are indistinguishable for case $_{1}$ and case $_{2}$. . . . . . . . . . . 13

12 Vapor curves for the three species considered (left), and cooling curve of the system (right). . 14

13 Normalized mass distributions for case $_{1}$ at different times. . . . . . . . . . . . 15

14 Normalized mass distribution at time 10 seconds for case $_{1}$ (above) and case $_{2}$ (below). . . . 15

15 Variation with radius of the ratio of normalized masses for different pairs of species for case $_{1}$ (above) and case $_{2}$ (below). Indicated are approximations to the linear coefficient

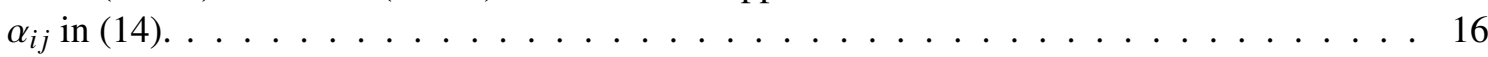





\title{
ACKNOWLEDGMENTS
}

This work was funded by the Office of Nuclear Detonation Detection - Forensics Program within the U. S. Department of Energy's National Nuclear Security Administration as part of the Water-Surface-Burst Fallout Model project.

\begin{abstract}
The development of chemical fractionation in particles condensing from a gas is described in terms of the intrinsic properties of the components and their mutual interaction through the nucleation, growth, and agglomeration processes. Possible ranges in the degree of fractionation are discussed in terms of a simple three-species configuration, and an example is given of the numerical description of this phenomenon.
\end{abstract}

\section{INTRODUCTION}

In multicomponent systems where particles* condense from a gas, the number and mass distributions as a function of particle size are the result of the time-evolving interaction of different physical processes. In some circumstances, the ratios of component masses are found to vary between particle samples and to differ from those of the system as a whole. The understanding of the mechanisms that cause this fractionation phenomenon can provide information on the particle formation history and plays an important role in the study of naturally occurring (Colzi et al. [2018]) and man-made (Freiling and Ballou [1962]) systems.

Fractionation has its origin in the differences in the condensation times of the gaseous species and the continuously evolving condensed phase. On the one hand, the vapor curve and concentration of a species in a gas mixture determine its supersaturation as a function of temperature. At the same time, vapor pressure gradients -the forces driving the mass fluxes between the condensed and gas phases- depend on the characteristics of the substrate onto which condensation is occurring. In particular, size, solubility, lattice compatibility, and chemical reactivity can be modifying factors of the saturation vapor pressure over the surface of a particle. As a result, the rate of condensation or evaporation is also a function of the condensed phase at a given time and can be altered by changes to the particle population by processes such as agglomeration, precipitation, or entrainment.

Here we focus on the evolution of a closed system where initially all materials are in gaseous form. We will assume that the conditions in the system are spatially uniform so that we can talk of a single (also called homogeneous by Kottler [1952]) particle population. As initially there is no substrate on which to condense, the formation of the new phase involves the need to overcome the energy barrier associated with the creation of the interface. This homogeneous nucleation process usually requires substantial supersaturation levels and results in a burst of particle production around a critical radius, which can be determined from energy considerations (Farley [1952]). Once a condensed phase exists, it is usually more energetically favorable for a vapor to condense on this substrate rather than to create new particles.

Apart from the nucleation and condensation (growth) mechanisms, collision and coalescence among particles can have a profound effect on the evolution of their population (Berry and Reinhardt [1974a,b]). The efficacy of this mechanism depends on the temperature, the velocity field interacting with the particles,

\footnotetext{
${ }^{*}$ We will also use the term particle to refer to droplets.
} 


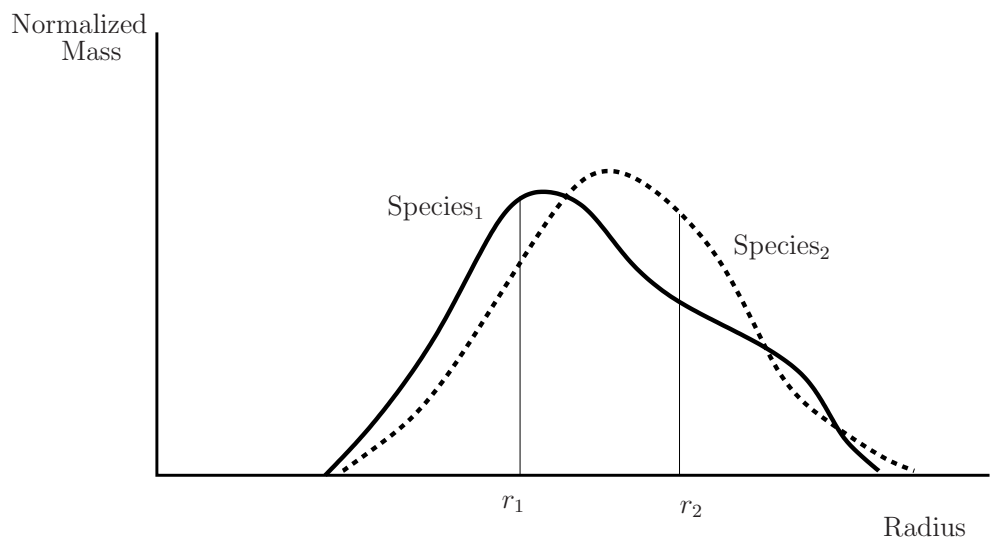

Figure 1. Differences in the normalized mass distribution of components of a particle population are an indication of fractionation.

and their size distribution and composition. Its overall effect is to induce internal mixing and to reduce the compositional differences among particles of different sizes.

A way to visualize the presence of fractionation in the particle population is to plot the normalized mass distribution of each component as a function of particle size. We will assume that the particles are spherical and their size can be described by their radius. As shown in Figure 1, the differences between these curves are an indication of variations in the mass ratios between components for different particle sizes. For example, samples consisting of particles with radius $r_{1}$ will show a mass ratio of species $_{1}$ to species $s_{2}$ that is larger than that in a sample of particles with radius $r_{2}$.

In this report we describe how these differences can arise as a combination of intrinsic properties of the species and the different physical processes responsible for the formation of the condensed phase. We exemplify this with a simple three-component system, in which one of the species can be identified as the most abundant and preponderant in determining the particle size distribution. This configuration is described qualitatively in Section 3 and with the results from a numerical model in Section 5. Section 4 discusses a means to quantify the fractionation characteristics of two components of the system when their mass distributions are lognormal. 


\section{SUBSTRATE DEPENDENCE}

A natural length scale for the condensation process is the mean free path of the vapor molecules in the gas. During growth, the dependence of the fluxes of mass on the radius of the particles can be expressed as a function of the Knudsen number, $K n$, which is the ratio of the mean free path to the particle radius. For $K n \ll 1$, the mass transport is dominated by diffusion and the mass flux (mass deposited per unit area and time) is inversely proportional to the radius of the particle. In the limit $K n \gg 1$, a molecular kinetics approach applies, and the flux is independent of particle size (Moresco [2020]).

Although there are analytical representations for the fluxes in those two limits, this is not the case for intermediate values of $K n$, and several approaches have been proposed that provide a continuous description as a function of $\mathrm{Kn}$. An example of this is shown in Figure 2 for the case of a system consisting of $\mathrm{FeO}$ vapor in air using the model by Gyarmathy [1982], where the molecular kinetics limit applies approximately for particles with radii under $10 \mu \mathrm{m}$ or with $K n \gtrsim 100$.

The vapor pressure of the condensing substance on the surface of a particle is a function of its ability to form a solution with the condensed phase. For a given level of supersaturation, this can result in a diminution of the vapor pressure on the surface of the particle and an enhancement of the condensation mass flux. When the condensing substance is at low concentrations in the condensed phase, its vapor pressure can be described by Henry's law, having a value proportional to its molar fraction in the solution (Atkins [1986]). As condensation progresses, this molar fraction will increase in smaller particles faster, leading to higher vapor pressures at the interface and favoring condensation into larger particles. Although, the distribution of mass condensed per unit time will be proportional to the distribution of particle surface, the variation of the vapor pressure with molar fraction can lead to mass distributions that with time become increasingly proportional to particle volumes. ${ }^{\dagger}$

As a result of surface tension, the vapor pressure on the surface of a particle is also affected by its radius through the Kelvin effect (Kelvin [1870], Moresco [2020]). This is particularly important in the type of system studied here, where the bulk of the particle population has radii in the submicron range, although the applicability of this classical law to nanometer-sized particles and the determination of an effective surface tension is still an area of active research (Kwon et al. [2018]).

Other processes affecting the particle population are collision and coalescence. Particles can be brought to the same region of space because of variations in the local velocity field or because of their different response to acceleration as a result of their inertia. Once in the proximity of each other, two particles may collide and coalesce, depending on their characteristics. This is usually described by means of a collection efficiency (Mason [1971]) that quantifies the probability that two particles will merge into one, once they are driven to the same region of space.

In a probabilistic description of the collision processes, the kernel $V\left(r_{1}, r_{2}\right)$ represents the probability that a particle with radius $r_{1}$ will collide with a particle of radius $r_{2}$, and the number of collisions per unit time and volume can be written $V\left(r_{1}, r_{2}\right) n_{1} n_{2}$, where $n_{1}$ and $n_{2}$ represent the volume density of particles with radius $r_{1}$ and $r_{2}$, respectively (Saffman and Turner [1956]). For submicron-sized particles, collisions are driven by Brownian motion, whereas for larger particles the dominant contribution to the kernel originates in gravitational settling (Seinfeld and Pandis [1997]).

In Figure 3 we employed a description of the collision kernel that takes into account these two mechanisms

\footnotetext{
${ }^{\dagger}$ This situation will be altered if diffusion in the condensed phase is slow or the substrate solidifies.
} 

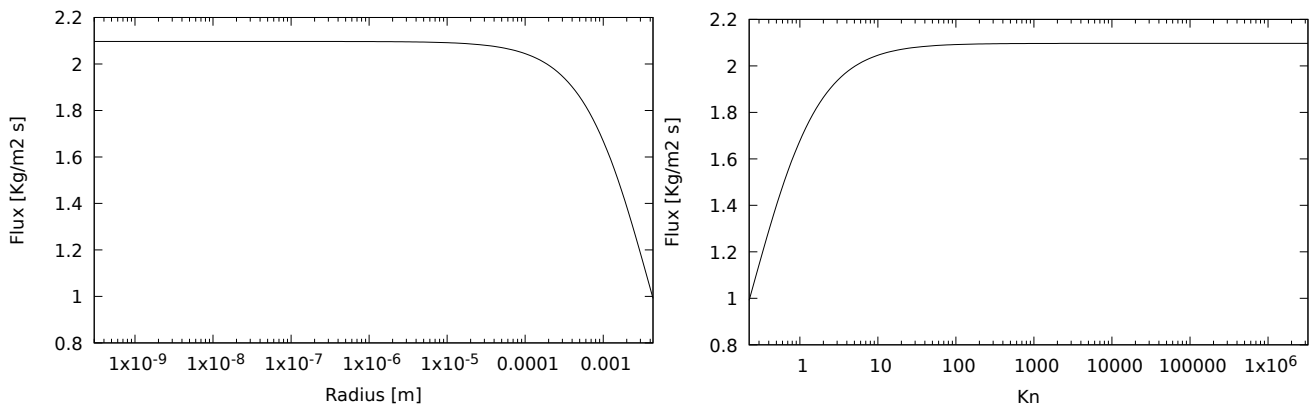

Figure 2. Mass fluxes as a function of particle radius and Knudsen number $(K n)$ for a FeO vapor in air at $2,000 \mathrm{~K}$.

and turbulence (Moresco [2020], Dodin and Elperin [2002]), and we show its dependence on the size of the particles. The kernel tends to have a local minimum when both particles have radii of the same order, with the minimum value occurring when the two radii are close to a micrometer. ${ }^{\ddagger}$ This is compatible with observations that systems condensing from the gas phase tend to show particle size distributions contained within the submicron range (Seinfeld and Pandis [1997]). For the configuration considered here, we can then expect the growth process to be driven by mass fluxes that are proportional to the area of the particles, and the coagulation interactions to be dominated by Brownian motion.
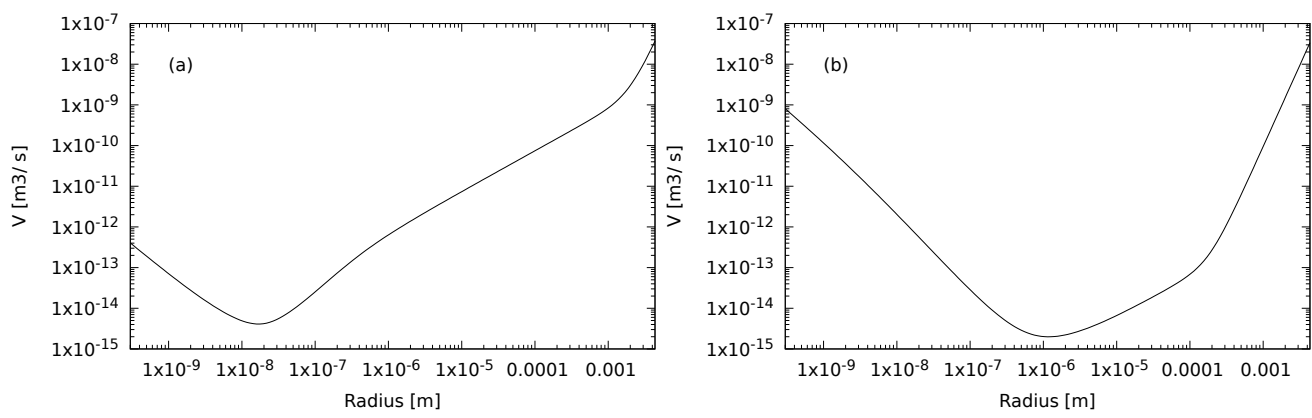

Figure 3. Magnitude of the collision kernel for particles with density $5.8 \mathrm{~g} / \mathrm{cm}^{3}$ in air at $2,000 \mathrm{~K} \mathrm{of}$ (a) a particle of radius $10^{-8} \mathbf{m}$ and (b) a particle with radius $10^{-6} \mathbf{m}$.

\footnotetext{
This is related to their Stokes relaxation time being of the same order as the characteristic time of the turbulent velocity field (Moresco [2020]).
} 


\section{EVOLUTION OF THE CONDENSED PHASE}

We will denote by $D_{n}(r)$ the normalized particle number distribution, where $D_{n}(r) d r$ denotes the fraction of the total number of particles with radii between $r$ and $r+d r$ and satisfying

$$
\int_{0}^{\infty} D_{n}(r) d r=1
$$

Different moments of the particle size distributions can be used to understand the composition characteristics of the condensed phase. For a distribution $D_{0}$, its moment distribution of ordinal $k$ corresponds to

$$
D_{k}(r)=\zeta_{k} r^{k} D_{0}(r)
$$

where $\zeta_{k}$ is a normalization constant. In the same manner, we can define distributions for the radius $D_{r}$, surface area $D_{s}$, and volume $D_{v}$ of the particles that satisfy

$$
\begin{aligned}
& D_{r}(r)=\zeta_{r} r D_{n}(r), \\
& D_{s}(r)=\zeta_{s} r^{2} D_{n}(r), \text { and } \\
& D_{v}(r)=\zeta_{v} r^{3} D_{n}(r) .
\end{aligned}
$$

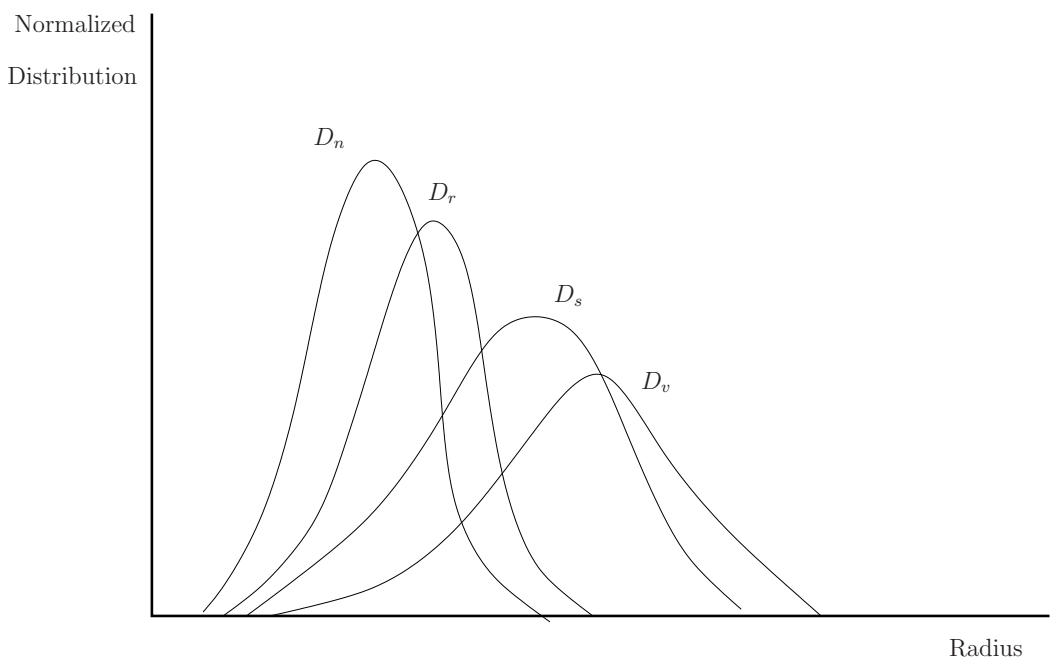

Figure 4. Schematic representation of the number, radius, surface and volume distributions for a particle population (not to scale).

The relation between the different distributions is shown qualitatively in Figure 4, which illustrates that the mean of the distributions tends to increase with the ordinal of the moment. As described below, depending on the characteristics of the particle population, the mass distribution of condensing substances can approximately follow one or another of these moment distributions, potentially giving rise to fractionation.

We now describe how the combined action of the nucleation, growth, and agglomeration processes can lead to the fractionation of the condensing species in the particle population. For simplicity, we restrict 
ourselves to a three-component system, in which one of the species is in larger proportion than the rest and constitutes the matrix of the condensed phase. We will call this species $B$ and the other two species $A$ and $C$. We assume that initially the system is at high enough temperature for all the species to be undersaturated in the vapor phase, and that it cools down monotonically with time. The properties of the species are taken to be such that they condense separately, in the sense that there are three non-overlapping time periods during which the mass flux between phases of one of the species is orders of magnitude larger than that of the other two. We will take the order of condensation to be first $A$, then $B$, and finally $C$. This configuration is usually described by saying that $C$ is more volatile than $B$, which in turn is more volatile than $A .{ }^{\S}$ Taking the matrix component as reference, it could also be said that $A$ and $C$ are, respectively, the refractory and volatile components of the system.

Our description will be in terms of limit cases that constitute bounds for real configurations. When we describe individual particle characteristics, they correspond to averages for the actual composition distributions. We take the nucleation, growth, and agglomeration processes to be the determining mechanisms of the rate of particle formation, with other processes occurring at much faster time scales. In real systems this will not necessarily be the case, and diffusion rates in the condensed phase could be limiting factors of the condensation process (Freiling [1969], Weisz et al. [2018]). In those cases, the assumption made here -that two miscible materials will always be in a state of complete dissolution-will not be valid. The mass distributions observed in real particle samples can then be expected to be intermediate to those presented here.

Note, the relation between a given species in the gas phase and the substrate is important mainly during its condensation period. For example, two species may be miscible at high temperatures but become separated into two distinct phases as the system cools down. If condensation has been completed under high miscibility conditions, the subsequent changes in the mass distributions within the particles will not have an effect on fractionation characteristics, because what are relevant for the latter are the total masses of each species in the particle.
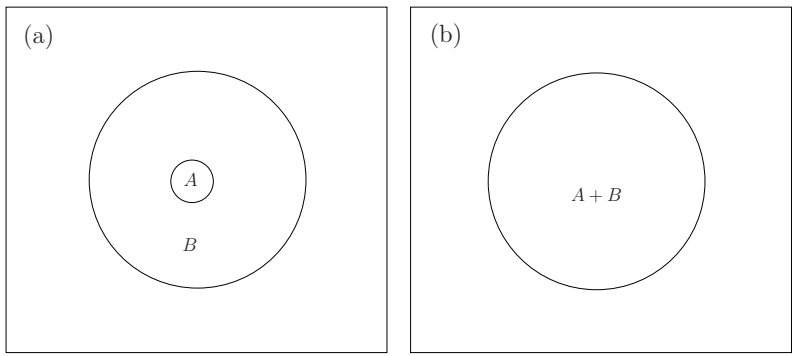

Figure 5. Condensation of $B$ on $A$ particles when the substances are not miscible (a) and when they form a solution (b).

As the system cools down, we will now assume that species $A$ forms the initial particle distribution by nucleation. This usually results in the creation, in a short period of time, of a large number of particles narrowly distributed around a radius of the order of nanometers. Once a substrate exists, condensation is driven by further growth of the existing particles. Except at high cooling rates, vapor depletion by growth will cause the supersaturation levels to rapidly decrease below the threshold required for nucleation, and the total particle number may simultaneously decrease as a result of the agglomeration process.

\footnotetext{
${ }^{\S}$ Here we say that a species is more volatile than another if it reaches supersaturation at a lower temperature, for the same vapor density.
} 
When material $B$ reaches supersaturation, it will usually be energetically more favorable for it to condense on the existing particles made of material $A$. Depending on the characteristics of the two species, this may result in the surface deposition of species $B$ (Figure 5[a]) or the formation of solution droplets (Fig. 5[b]). As time progresses, and because of its higher abundance in the vapor phase, species $B$ eventually will become the dominant component of the particles, and its mass distribution will be proportional to the particle volume distribution. If $A$ is soluble in $B$, its mass distribution will also be volumetric, and there will be little fractionation between the two species (Figure 6[a]). If

If the two species are immiscible, the final mass distribution of $A$ will largely depend on the properties of the coagulation processes. If there are few collisions among the particles, the population will show particle characteristics as depicted in Figure 7(a). As $B$ becomes the dominant component, progressively the characteristics of the initial population formed by $A$ will have less of an influence on the particle characteristics. In the limit where the mass of $A$ is independent of particle size, its distribution will depend only on the particle number density; that is, the amount of $A$ in each size class will depend only on the number of particles in that size class. Consequently, apart from a normalization constant, the mass distribution of $A$ will be identical to the particle number distribution, whereas that of $B$ will follow the distribution of particle volume (Figure 6[b]). On the other hand, when agglomeration is strong, composition patterns as shown in Figure 7(b) will be more abundant, where the mass of $A$ is approximately proportional to the volume of the particles (Figure 6[a]).
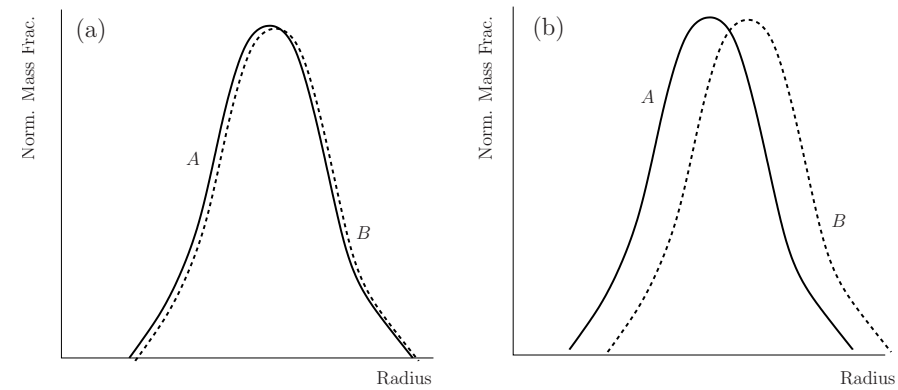

Figure 6. Schematic representation of the mass distributions of species $A$ and $B$ when they are miscible (a) and when they are not (b).

Later, when species $C$ reaches supersaturation, it will condense on the existing particles formed of $A$ and $B$. Depending on the ability of species $C$ to dissolve in the particles, at the end of the condensation period the condensate will show characteristics similar to those in Figure 8. In the case of Figure 8(a), the mass distributions of $A, C$, and $B$ follow the particle number, surface, and volume distributions, respectively, as shown schematically in Figure 9(a). In Figure 8(b), the mass distributions of $A$ and $B$ are both volumetric, but that of $C$ is proportional to the surface of the particles, resulting in mass distributions like those in Figure 9(b). In Figure 8(c), the mass of $A$ is proportional to the number of particles, but those of $B$ and $C$ are proportional to their volume, shielding the distributions shown in Figure 9(c). Finally, in the case of Figure 8(d) all species are distributed volumetrically, giving mass distributions qualitatively similar to Figure 9(d).

If $A$ and $B$ are able to form a solution, the mass distribution of $C$ will depend on whether it is able to dissolve in it, as shown in Figure 10. In the case where all substances are miscible (Figure 10[a]), the final distribution of the masses of the three species will be proportional to the volume of the particles, giving a

\footnotetext{
${ }^{\text {II }}$ We also note that this configuration would also be observed if $B$ were to condense before $A$.
} 

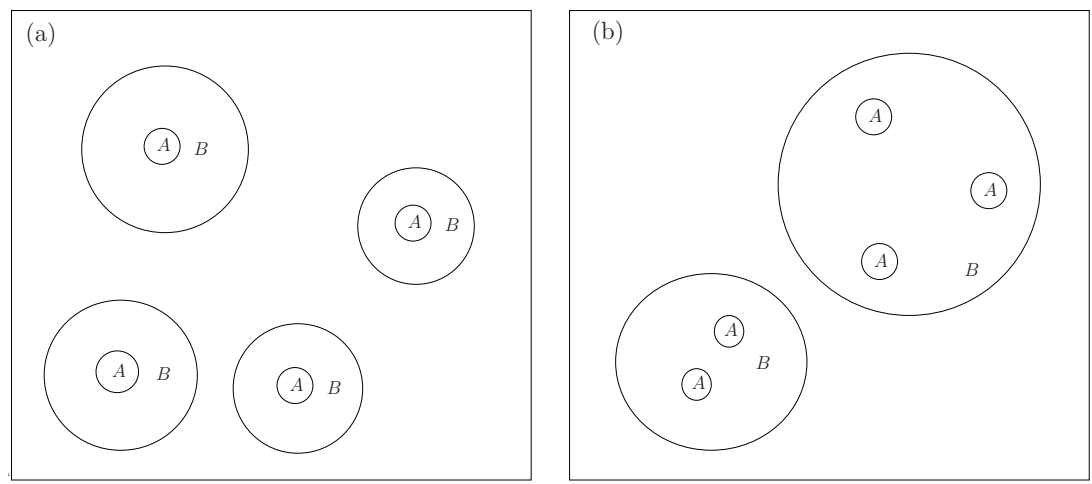

Figure 7. Particle characteristics when $A$ and $B$ are immiscible when coagulation is weak (a) and when it is strong (b).

low degree of fractionation (Figure 10[c]). If species $C$ is insoluble in the $A+B$ complex, it will be distributed in a form proportional to the surface of the particles (Figure 10[b]), and the mass distributions will correspond to Figure 10(d).
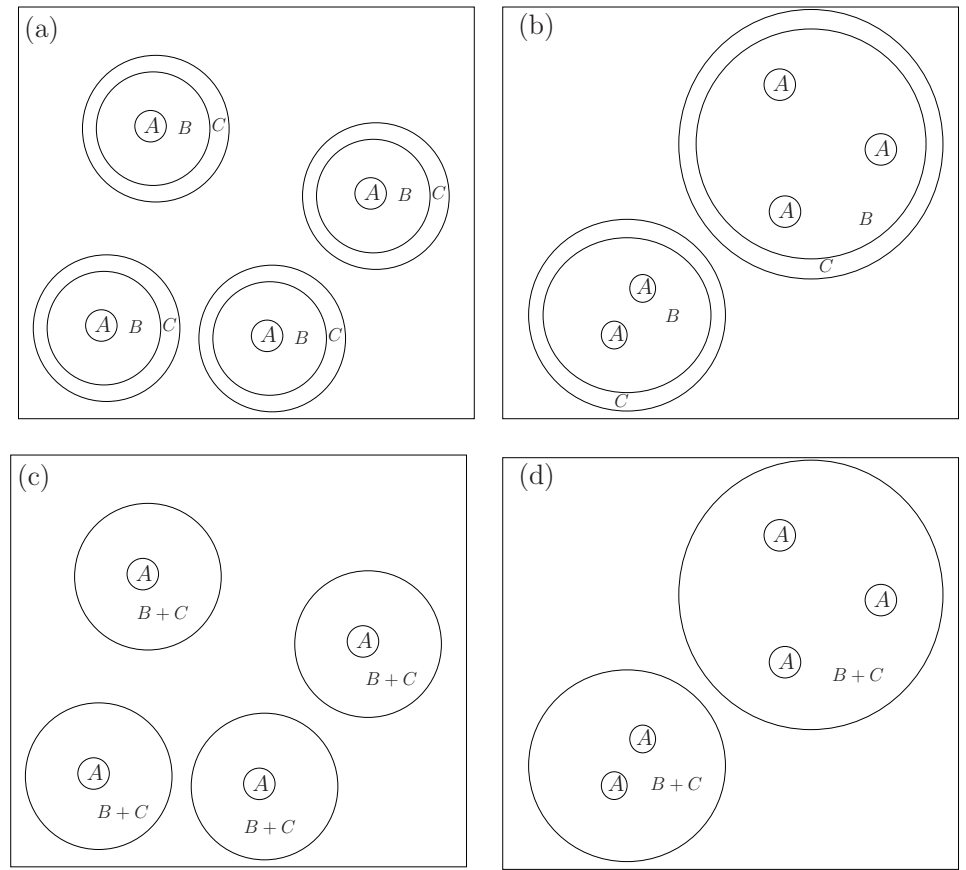

Figure 8. Variations in particle characteristics as a function of the solubility of $C$ in $B$, when $A$ and $B$ are not miscible. 

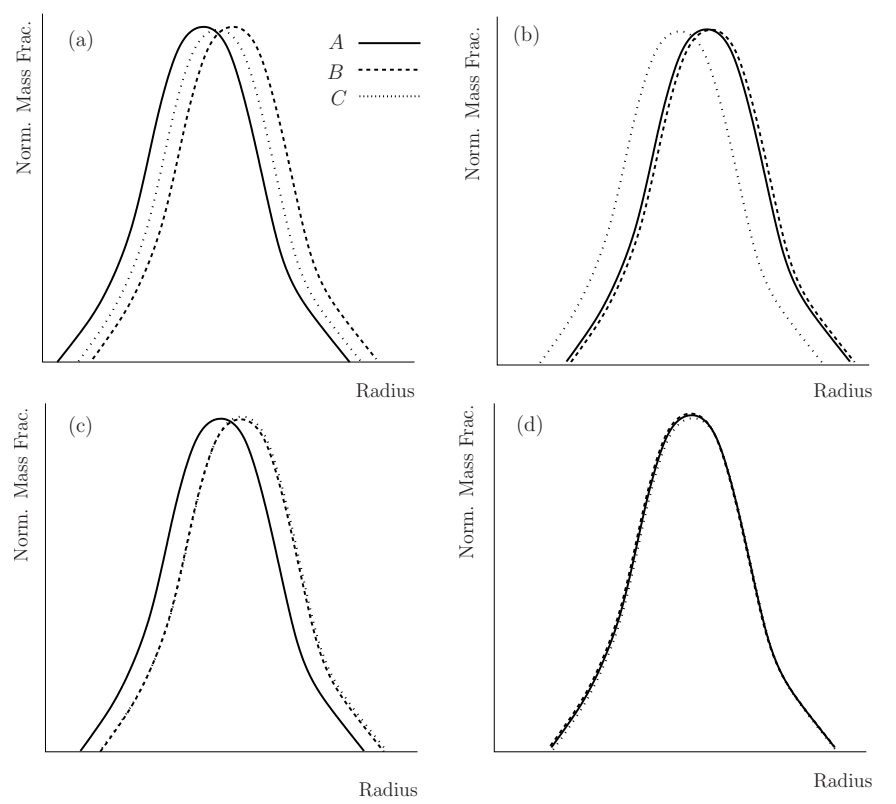

Figure 9. Schematic representation of the species mass distributions corresponding to Figure 8.
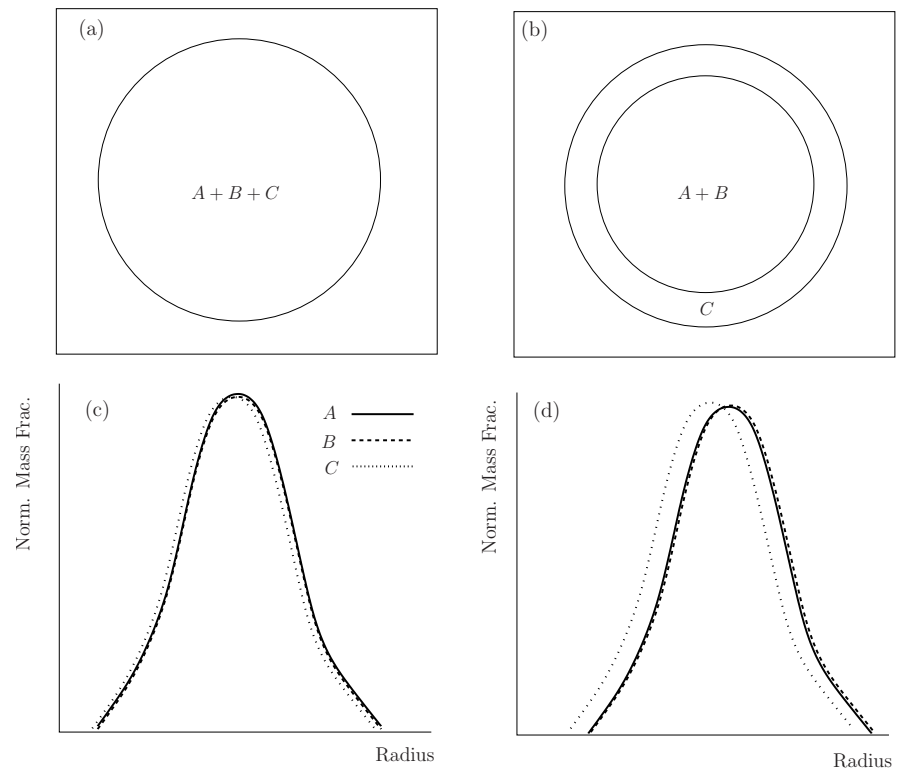

Figure 10. Particle characteristics and species mass distributions as a function of the solubility of $C$ in $B$, when $A$ and $B$ are miscible. 



\section{MASS RATIOS}

A quantitative measure of the degree of fractionation can be obtained if a functional form is assumed for the particle size distribution. Although there is no rigorous proof that particles formed from a gas phase will follow a lognormal distribution, there is experimental and, for simplified configurations, analytical evidence, that in general this type of distribution is among the best approximations (Kottler [1950a,b], Stewart [1956], Nathans et al. [1969], Buhrman and Granqvist [1976], Granqvist and Buhrman [1976], Kiss et al. [1999]).

A lognormal number size distribution can then be written as

$$
D_{n}(\ln r)=\frac{1}{\sqrt{2 \pi} \ln \sigma} \exp \left[-\frac{(\ln r-\ln \bar{r})^{2}}{2 \ln ^{2} \sigma}\right],
$$

where $\bar{r}$ is the median radius, and $\sigma$ is the geometric standard deviation.

A property of this type of distribution is that its moment distributions are also lognormal with the same $\sigma$. This can be seen for the $k^{\text {th }}$ moment:

$$
r^{k} D_{n}(\ln r)=\frac{\zeta_{k}}{\sqrt{2 \pi} \ln \sigma} \exp \left[-\frac{(\ln r-\ln \bar{r})^{2}}{2 \ln ^{2} \sigma}\right] \exp (k \ln r),
$$

where $\zeta_{k}$ is a normalization constant from equations (3) to (5). We then obtain

$$
r^{k} D_{n}(\ln r)=\frac{\zeta_{k}}{\sqrt{2 \pi} \ln \sigma} \exp \left(\frac{k^{2} \ln ^{2} \sigma}{2}\right) \exp \left[-\frac{\left(\ln r-\ln \bar{r}^{\prime}\right)^{2}}{2 \ln ^{2} \sigma}\right] \exp (k \ln \bar{r}),
$$

where

$$
\ln \bar{r}^{\prime}=\ln \bar{r}+k \ln ^{2} \sigma .
$$

This applies both for integer and real $k$, and we can then talk of fractional moments of the number distribution that are also lognormal with the same $\sigma$.

We will denote by $\mu_{i}$ the normalized mass distributions for species $i$ with median radius $\bar{r}_{i}$, and we will assume that the mass distributions of two components of the particles have the same geometric standard deviation,

$$
\begin{aligned}
& \mu_{i}(\ln r)=\frac{\zeta_{i}}{\sqrt{2 \pi} \ln \sigma} \exp \left[-\frac{\left(\ln r-\ln \bar{r}_{i}\right)^{2}}{2 \ln ^{2} \sigma}\right], \text { and } \\
& \mu_{j}(\ln r)=\frac{\zeta_{j}}{\sqrt{2 \pi} \ln \sigma} \exp \left[-\frac{\left(\ln r-\ln \bar{r}_{j}\right)^{2}}{2 \ln ^{2} \sigma}\right]
\end{aligned}
$$

If we define $\Delta_{i j}=\ln \bar{r}_{i}-\ln \bar{r}_{j}$, the distribution of mass ratios can then be written

$$
\frac{\mu_{i}(\ln r)}{\mu_{j}(\ln r)}=\frac{\zeta_{i}}{\zeta_{j}} \exp \left(\frac{\Delta_{i j} \ln r}{\ln ^{2} \sigma}\right) \exp \left[\frac{\Delta_{i j}}{2 \ln ^{2} \sigma}\left(\Delta_{i j}-2 \ln \bar{r}_{i}\right)\right]=A_{i j} r^{\alpha_{i j}},
$$

where

$$
\alpha_{i j}=\Delta_{i j} / \ln ^{2} \sigma, \quad A_{i j}=\zeta_{i} / \zeta_{j} \exp \left[\left(\Delta_{i j}^{2}-2 \ln \bar{r}_{i} \Delta_{i j}\right) / 2 \ln ^{2} \sigma\right]
$$


In this description of $\mu_{j}(\ln r)$ as a moment distribution of $\mu_{i}(\ln r), \alpha_{i j}$ plays the same role as $k$ in (9). We then have a linear relation between the logarithm of the radius and mass ratios,

$$
\ln \left(\frac{\mu_{i}}{\mu_{j}}\right)=\alpha_{i j} \ln (r)+\ln \left(A_{i j}\right)
$$

In the simple configuration analyzed in Section 3, the mass distributions of the species were described as following one of the moments of the number distribution (e.g., as being proportional to particle surfaces or volumes). In the case those distribution are lognormal, we can use the value of $\alpha_{i j}$ in (14) as a measure of the degree of fractionation between species $i$ and $j$,

For the limit cases discussed in Section 3, and shown in Figures 9 and 10, the values of $\alpha_{i j}$ are listed in Table 1." The maximum value in magnitude (i.e., 3) occurs when one of the species is distributed volumetrically, whereas the other follows the particle number distribution. On the other hand, the minimum value, $\alpha_{i j} \approx 0$ corresponds to the case when there is little fractionation between the two species.

Furthermore, Figures 9(d) and 10(c) are examples of particles with similar fractionation characteristics but different internal species distributions (i.e, $8[\mathrm{~d}]$ and $10[\mathrm{a}]$ ).

Table 1. Value of the coefficient $\alpha_{i j}$ in the cases considered in Section 3.

\begin{tabular}{cccc}
\hline \hline Figure & $\alpha_{A B}$ & $\alpha_{C B}$ & $\alpha_{A C}$ \\
\hline $9(\mathrm{a})$ & -3 & -1 & -2 \\
$9(\mathrm{~b})$ & 0 & -1 & 1 \\
$9(\mathrm{c})$ & -3 & 0 & -3 \\
$9(\mathrm{~d})$ & 0 & 0 & 0 \\
$10(\mathrm{c})$ & 0 & 0 & 0 \\
$10(\mathrm{~d})$ & 0 & -1 & 1 \\
\hline
\end{tabular}

\footnotetext{
"The differences in sign arise from the order of the species in the definition of $\alpha_{i j}$.
} 


\section{NUMERICAL REPRESENTATION}

Analytical descriptions of the fractionation characteristics in nuclear debris have been developed from sample measurements during the period of the testing of nuclear weapons (Freiling [1961]). They are physically realistic and computationally economic and are widely implemented in the operational codes in use today. Relating those fractionation characteristics to the processes responsible for the formation of the debris particles is substantially more complex and can usually only be achieved by means of numerical descriptions (Moresco et al. [2014]). To be physically relevant, the models adopted need to capture the essence of the nucleation, growth, and agglomeration processes and must be able to describe particle characteristics with a degree of accuracy that resolves the changes induced by fractionation. We show here an example of such a calculation.

The method employed is based on a sectional discretization of the particle size distribution, where particles are grouped into a finite number of sections or bins according to their size (Gelbard et al. [1980]). Within each section, we further assume that all the particles have the same size and composition, and no information on their internal structure is maintained (Moresco [2020]).

We model a system of three species with the same characteristic as those described in Section 3. We carried out two calculations: case $_{1}$ using a collection efficiency with value one, and case $_{2}$ in which the collection efficiency is reduced to $10^{-4}$ after 1 second, that is, well after species $A$ has finished condensing (Figure 11).
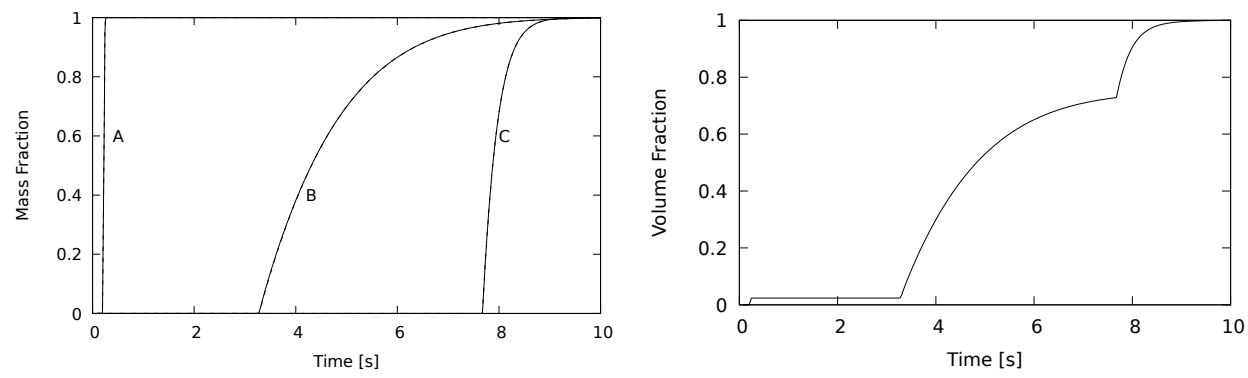

Figure 11. Fraction of the total mass in the condensed phase as a function of time for each species (left), and volume fraction of the condensed phase normalized for its value at time $\mathbf{1 0}$ seconds (right). These curves are indistinguishable for $\operatorname{case}_{1}$ and $\operatorname{case}_{2}$.

We further assume the three species are immiscible, and we focus on the effect of the agglomeration mechanism on the final mass distributions (i.e., roughly on the differences between configurations 8[a] and $8[\mathrm{~b}]$ ). For this, we use three species with the vapor curves shown in Figure 12(a). The initial system was taken to be air at $5,300 \mathrm{~K}$ and the species concentrations to be $A=1.4 \times 10^{-3} \mathrm{~kg} / \mathrm{m}^{3}, B=4 \times 10^{-2} \mathrm{~kg} / \mathrm{m}^{3}$ and $C=1.6 \times 10^{-2} \mathrm{~kg} / \mathrm{m}^{3}$ to represent the variations in concentration described in Section 3 . The system was cooled at constant pressure, without exchanging mass with its exterior, according to the curve shown in Figure 12(b). To reduce the number of parameters we took the molar masses and densities in the condensed phase to be the same for the three species (i.e., $100 \mathrm{~g}$ and $5.8 \mathrm{~g} / \mathrm{cm}^{3}$ ).

Figure 11 shows that for this configuration the species condense during three clearly separated periods. The mass distributions at different times for case $_{1}$ are shown in Figure 13. Species $A$ is in the smallest fraction and due to the fast cooling rate condenses mainly by nucleation, with the further action of agglomeration 
leading by 1 second to the mass distribution shown in Figure 13. Initially the distribution of $B$ is confined to the surface of the particles ( 3.3 seconds), but as it becomes the major component of the particles, the distribution evolves to one where the masses of both species are proportional to the volume of the particles (3.8 seconds). This is different in case $_{2}$, where the coagulation is reduced and there is a more marked difference in the mass distribution of $A$ and $B$. Since coagulation is not completely absent in this configuration, we can expect particle characteristics somewhere between those in Figures 7(a) and 7(b).

In both calculations, the condensation of species $C$ starts at around 7.7 seconds and initially it is concentrated on the surface of the particles, but because it represents around $30 \%$ of the condensed volume, its final distribution is intermediate between surface and volumetric. In terms of relative mass distributions, in case $_{1}$ the similarity between $A$ and $B$ implies that their relation to $C$ is also similar; however in case $_{2}$ the mass distributions of the three species are different. The mass distributions at 10 seconds for both calculations, when the three species have finished condensing, are shown in Figure 14.

To better quantify the relation between the mass distributions, in Figure 15 we plotted the ratios of normalized mass distributions for pairs of species as a function of particle radius. Although the calculated mass distributions are not expected to be exactly lognormal, and relation (14) would not be strictly valid, we also drew linear approximations to these curves and indicated their slopes. For case $_{1}$, we find $\alpha_{A B} \approx 0$ because both distributions are approximately volumetric, although relative to them the distribution of $C$ results in $0 \lesssim \alpha_{A C} \lesssim 1$ and $-1 \lesssim \alpha_{C B} \lesssim 0$, which agrees with its distribution being between surface and volumetric.

The situation is more complex in case $_{2}$. Now the reduced action of the coagulation mechanism results in a higher number of smaller particles by the time condensation finishes (Figure 14 [below]). Because for spherical particles the surface to volume ratio is inversely proportional to the radius, the distribution of $C$ is now closer to volumetric than in case $_{1}$, and $\alpha_{C B}$ is closer to zero. As expected, $\alpha_{A B}$ is within the range $-3 \lesssim \alpha_{A B} \lesssim 0$, with the mass distribution of $A$ being closer to surface rather than volumetric.

Although the order and temperature ranges in which the species condense is approximately the same for case $_{1}$ and case $_{2}$, the resulting relative mass distributions are different. If the classification of species in terms of volatile or refractory were to be based on these distributions, different conclusions would be drawn from the two calculations. This illustrates that the observed patterns of condensation are the result of multiple processes and cannot be predicted solely on the basis of intrinsic properties of the species, such as vapor curves.
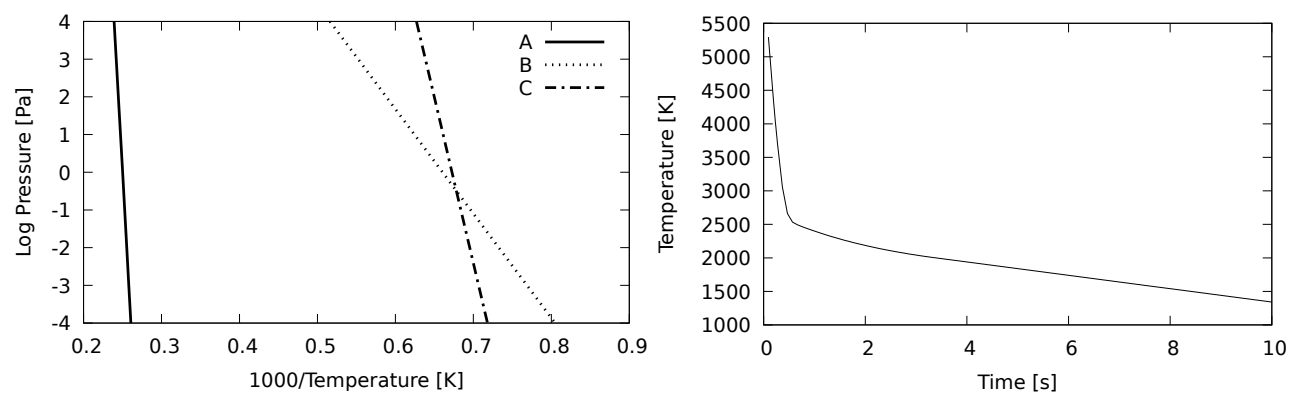

Figure 12. Vapor curves for the three species considered (left), and cooling curve of the system (right). 

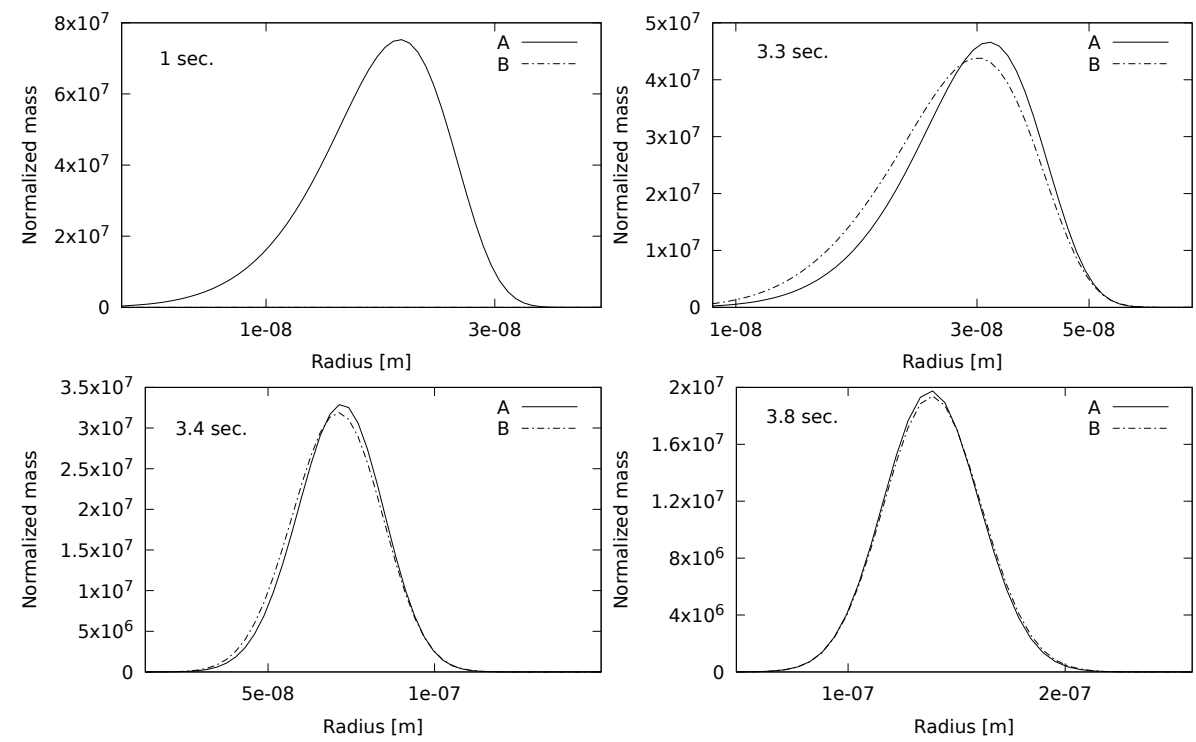

Figure 13. Normalized mass distributions for case $_{1}$ at different times.
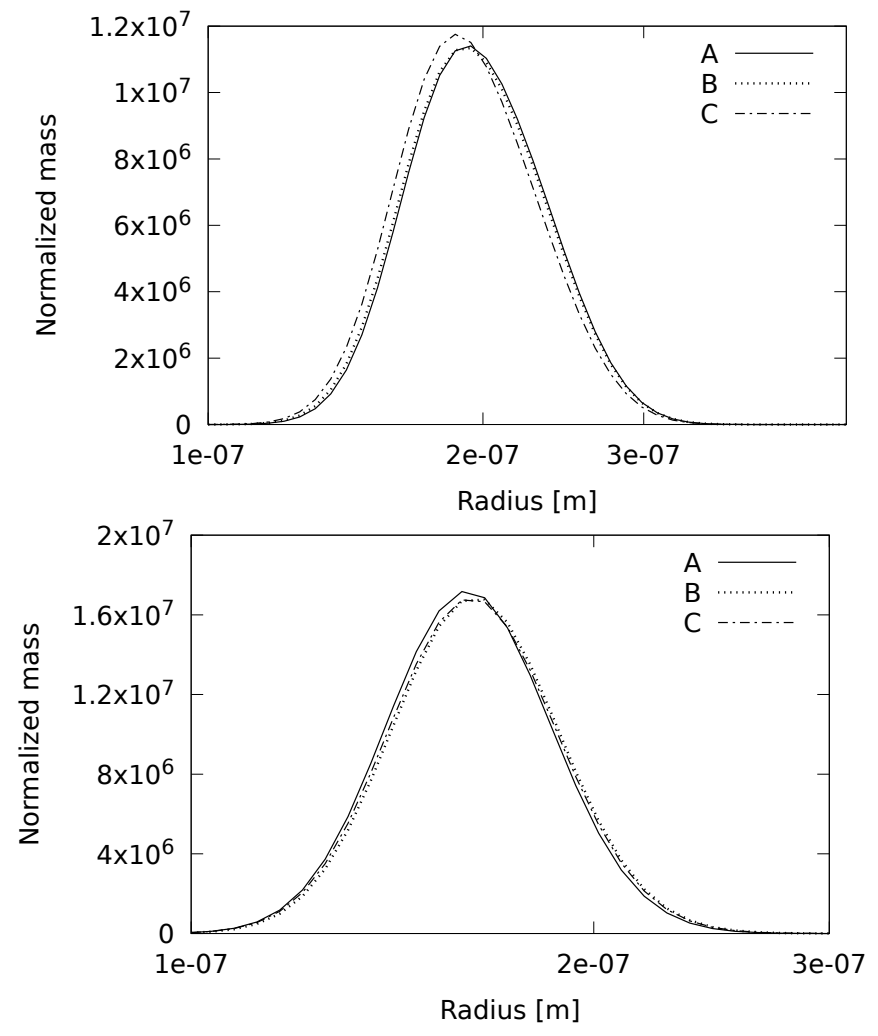

Figure 14. Normalized mass distribution at time 10 seconds for case $_{1}$ (above) and case $_{2}$ (below). 

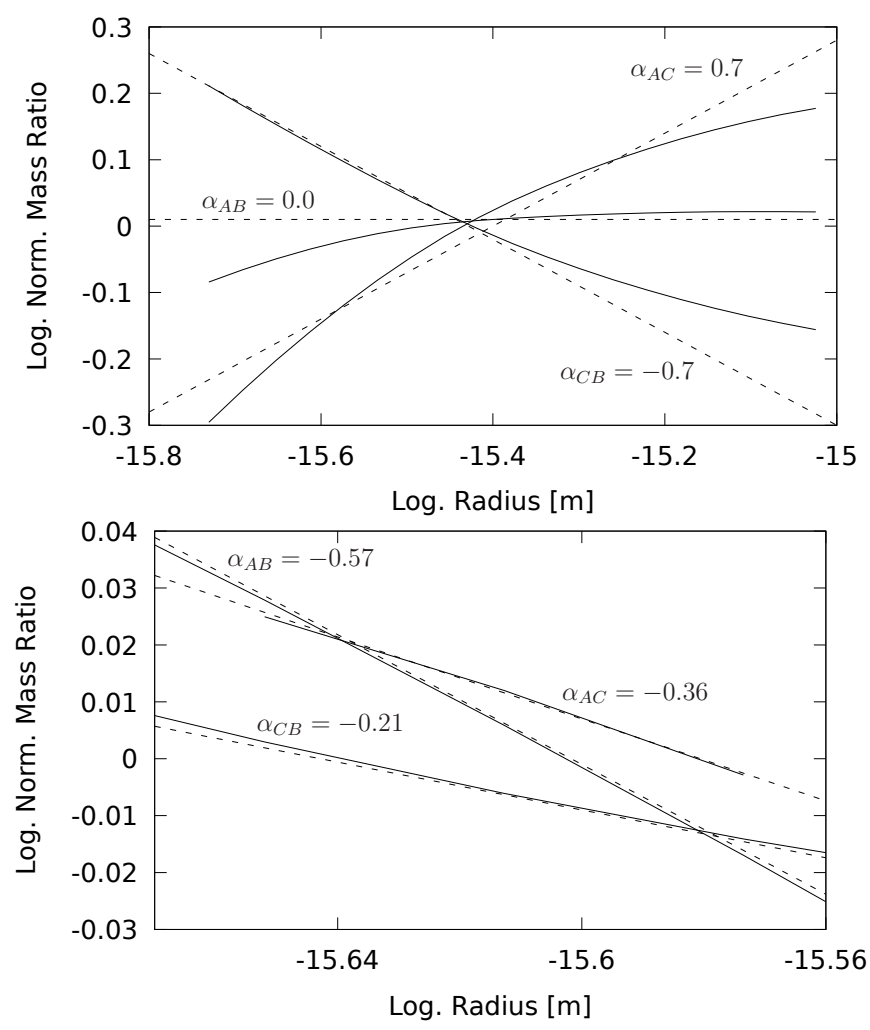

Figure 15. Variation with radius of the ratio of normalized masses for different pairs of species for case $_{1}$ (above) and case $_{2}$ (below). Indicated are approximations to the linear coefficient $\alpha_{i j}$ in (14). 


\section{CONCLUSIONS}

Variations in component mass ratios for different particle samples is a common occurrence in multicomponent systems condensing from the gas phase and arises from the intrinsic properties of the species involved and the continuously evolving substrate. The phenomenon of fractionation has received special attention within the study of the formation of radioactive debris after a nuclear detonation, where the ability to model the relative abundance of radionuclides in those particles has important consequences for the prediction of the spatial distribution of contamination and in the forensic study of the characteristics of the weapon (Freiling [1961, 1966]).

Air and ground debris samples after nuclear tests have been found to have marked variations in the nuclide mass distributions with particle size (Edvarson et al. [1959], Mackin et al. [1960], Adams et al. [1960], Benson et al. [1965], Mamuro et al. [1969]). Partially based on their thermodynamic properties, species that were enriched in the smaller particles were classified as volatile, whereas those present in larger proportion in the larger size ranges as refractory.** One of the earliest attempts at explaining why the species are distributed in this way was by Magee [1953], who postulated that refractory materials are responsible for the initial particle formation, initiated by nucleation, and that the turbulent flow field, plus the action of gravitational settling, cause the condensate, especially the larger particles, to separate from the volatile vapors, thus reducing their ability to act as a substrate for their condensation. Nevertheless, this interpretation does not explain the observed marked fractionation in particles with radii in the micrometer range and smaller (Mamuro et al. [1968], Moore et al. [1973]), which given their small inertia, are unlikely to precipitate from the gas flow during the time period condensation occurs.

Freiling [1963b] suggested accounting for the role of the particle matrix, and he postulated that the mass distributions of the minor components would follow different moment distributions of the particle population. His model predicts that the refractory materials will condense onto molten particles and will be volumetrically distributed in them, whereas by the time volatile components reach supersaturation, the particles will have solidified and the condensation will be restricted to their surfaces. This would correspond to the configuration in Figure 10(b), although with $B$ condensing earlier than $A$. Freiling recognized that in practical applications mass distributions will be found to be intermediate to those limit cases, and under the assumption of lognormal distributions, he proposed an empirical approach to predicting the fractionation properties based on the correlation of the linear fit coefficients $\alpha_{i j}$ in (14) between different pairs of nuclides (Freiling [1963a]).

In this work we built on Freiling's ideas and showed that the combined effects of nucleation, growth, and agglomeration need to be taken into account to better describe possible variations in fractionation characteristics. As a result of this analysis it should also become clearer that the vapor curves of individual species are not sufficient to predict the characteristics of their mass distribution in the particle population. The dependence of the condensation process on the available substrate introduces an interdependence among the different components that involves also their relative abundances and properties in the condensed phase. Also the characteristics of the gas flow, such as the intensity of turbulence and the cooling rate, play an important part, affecting quantities such as diffusion coefficients and collision kernels, and hence the rates of condensation and agglomeration.

As shown in the simple configuration considered here, the relation in the mass distributions of two minor

\footnotetext{
${ }^{* *}$ Because of the radioactive nature of these materials, nuclear debris fractionation tends to be described in terms of mass chains instead of single species.
} 
components of the system can vary if certain parameters of the configuration change. Thus certain species can migrate within the volatile and refractory classifications, depending on the conditions under which condensation takes place. The ability to understand and predict this type of behavior is crucial not only in the realm of nuclear forensics, but also in the study of particle production processes for industrial applications (Koch et al. [2005], Dvorský et al. [2013], Bunge et al. [2019]). With the example of a numerical calculation, we have also shown that it is possible to combine first principles models with current computational capabilities to describe the development of fractionation characteristics, thus reducing the need for empirical parameterization. 


\section{REFERENCES}

C. E. Adams, N. H. Farlow, and W. R. Schell. The compositions, structures and origins of radioactive fall-out particles. Geochimica et Cosmochimica Acta, 18:42-56, 1960.

P. W. Atkins. Physical chemistry. Oxford University Press, 1986.

P. Benson, C. E. Gleit, and L. Leventhal. Physical characteristics of single particles from high yield air bursts. In Proc. Second USAEC Symp. Radioactive Fallout from Nuclear Weapons Tests, pages 98-107, 1965.

E. X. Berry and R. L. Reinhardt. An analysis of cloud drop growth by collection: Part I. Double distributions. J. Atmos. Sci., 31:1814-1824, 1974a.

E. X. Berry and R. L. Reinhardt. An analysis of cloud drop growth by collection: Part II. Single initial distributions. J. Atmos. Sci., 31:1825-1831, 1974b.

R. A. Buhrman and C. G. Granqvist. Log-normal size distributions from magnetization measurements on small superconducting Al particles. J. Appl. Phys., 47:2220-2222, 1976.

A. Bunge, A. S. Porav, G. Borodi, T. Radu, A. Pîrnău, C. Berghian-Grosan, and R. Turcu. Correlation between synthesis parameters and properties of magnetite clusters prepared by solvothermal polyol method. J. Mater. Sci., 54:2853-2875, 2019.

L. Colzi, F. Fontani, P. Caselli, C. Ceccarelli, P. Hily-Blant, and L. Bizzocchi. Nitrogen and hydrogen fractionation in high-mass star-forming cores from observations of $\mathrm{HCN}$ and $\mathrm{HCN}^{*}$. AEEA, 609:A129, 2018.

Z. Dodin and T. Elperin. On the collision rate of particles in turbulent flow with gravity. Phys. Fluids, 14: 2921-2924, 2002.

R. Dvorský, J. Trojková, P. Praus, and J. Lun̆áček. Analysis of mechanisms of composite particles modification in liquid dispersions. Mater. Res. Bull., 48:2286-2288, 2013.

K. Edvarson, K. Löw, and J. Sisefsky. Fractionation phenomena in nuclear weapons debris. Nature, 184: 1771-1774, 1959.

F. J. M. Farley. The theory of the condensation of supersaturated ion-free vapour. Proc. Roy. Soc. A, 212: $530-542,1952$.

E. C. Freiling. Radionuclide fractionation in bomb debris. Science, 133:1991-1998, 1961.

E. C. Freiling. Fractionation III. Estimation of degree of fractionation and radionuclide partition for nuclear debris. Report USNRDL-TR-680, US Naval Radiological Defense Laboratory, 1963a.

E. C. Freiling. Theoretical basis for logarithmic correlations of fractionated radionuclide compositions. Science, 139:1058-1059, 1963b.

E. C. Freiling. Radionuclide fractionation in air-burst debris. Nature, 209:209-238, 1966.

E. C. Freiling. Mass-transfer mechanisms in source-term definition. Advan. Chem. Ser., 93:1-12, 1969. 
E. C. Freiling and N. E. Ballou. Nature of nuclear debris in sea-water. Nature, 195:1283-1287, 1962.

F. Gelbard, Y. Tambour, and J. H. Seinfeld. Sectional representations for simulating aerosol dynamics. J. Colloid Interface Sci., 76:541-556, 1980.

C. G. Granqvist and R. A. Buhrman. Ultrafine metal particles. J. Appl. Phys., 47:2200-2219, 1976.

G. Gyarmathy. The spherical droplet in gaseous carrier streams: Review and synthesis. Multiphase Sci. Tech., 1:99-279, 1982.

Lord Kelvin. On the equilibrium of vapour at a curved surface of a liquid. Proc. Roy. Soc. Edinb., 7:63-68, 1870 .

L. B. Kiss, J. Söderlund, G. A. Niklasson, and C. G. Granqvist. The real origin of lognormal size distributions of nanoparticles in vapor growth processes. Nanostruct. Mater., 12:327-332, 1999.

J. Koch, H. Lindner, A. von Bohlen, R. Hergenröder, and K. Niemax. Elemental fractionation of dielectric aerosols produced by near-infrared femtosecond laser ablation of silicate glasses. J. Anal. At. Spectrom., 20:901-906, 2005.

F. Kottler. The distribution of particle sizes. Part I. The facts. J. Franklin Inst., 250:339-356, 1950a.

F. Kottler. The distribution of particle sizes. Part II. The probability graphs. J. Franklin Inst., 250:419-441, $1950 \mathrm{~b}$.

F. Kottler. The logarithmico-normal distribution of partiucle sizes: Homogeneity and heterogeneity. $J$. Phys. Chem., 56:442-448, 1952.

S. Kwon, B. Kim, S. An, W. Lee, H. Kwak, and W. Jhe. Adhesive force measurement of steady-state water nano-meniscus: Effective surface tension at nonscale. Sci. Rep., 8:8462, 2018.

J. Mackin, P. Zigman, D. Love, D. Macdonald, and D. Sam. Radiochemical analysis of individual fall-out particles. J. Inorg. Nucl. Chem., 15:20-36, 1960.

J. L. Magee. Mechanisms of fractionation. Office Memorandum M-7140, Los Alamos National Laboratory, 1953.

T. Mamuro, T. Matsunami, and A. Fujita. Radionuclide fractionation in fallout particles from an air burst. Health Phys., 14:223-239, 1968.

T. Mamuro, T. Matsunami, A. Fujita, and K. Yoshikawa. Radionuclide fractionation in fallout particles from a land surface burst. J. Geophys. Res., 74:1374-1387, 1969.

B. J. Mason. The physics of clouds. Oxford University Press, 1971.

D. T. Moore, J. N. Beck, D. K. Miller, and P. K. Kuboda. Radioactive hot particles from the recent Chinese nuclear weapons tests. J. Geophys. Res., 78:7039-7050, 1973.

P. Moresco. Description of nucleation, growth and coagulation processes in the modeling of debris formation after a nuclear burst. Report ORNL/TM-2020/1694, Oak Ridge National Laboratory, 2020.

P. Moresco, T. E. Harris, and V. Jodoin. Vorticity generation by the instantaneous release of energy near a reflective boundary. Phys. Rev. E, 90:023002, 2014. 
M. W. Nathans, R. Thews, and I. J. Russell. The particle size distribution of nuclear cloud samples. Advan. Chem. Ser., 93:360-380, 1969.

G. Saffman and J. S. Turner. On the collision of drops in turbulent clouds. J. Fluid Mech., 1:16-30, 1956.

J. H. Seinfeld and S. N. Pandis. Atmospheric chemistry and physics: From air pollution to climate change. Wiley, 1997.

K. Stewart. The condensation of a vapour to an assembly of droplets or particles. Trans. Farad. Soc., 52: 161-173, 1956.

D. G. Weisz, B. Jacobsen, N. E. Marks, K. B. Knight, B. H. Isselhardt, and J. E. Matzel. Diffusive mass transport in agglomerated glassy fallout from a near-surface nuclear test. Geochimica et Cosmochimica Acta, 223:377-388, 2018. 
